\title{
The assembly and characterisation of two structurally distinct cattle MHC class I haplotypes point to the mechanisms driving diversity
}

\author{
John C. Schwartz ${ }^{1}$ John A. Hammond ${ }^{1}$
}

Received: 2 June 2015 / Accepted: 20 July 2015 / Published online: 31 July 2015

(C) The Author(s) 2015. This article is published with open access at Springerlink.com

\begin{abstract}
In cattle, there are six classical MHC class I genes that are variably present between different haplotypes. Almost all known haplotypes contain between one and three genes, with an allele of Gene 2 present on the vast majority. However, very little is known about the sequence and therefore structure and evolutionary history of this genomic region. To address this, we have refined the MHC class I region in the Hereford cattle genome assembly and sequenced a complete A14 haplotype from a homozygous Holstein. Comparison of the two haplotypes revealed extensive variation within the MHC class Ia region, but not within the flanking regions, with each gene contained within a conserved 63- to 68-kb sequence block. This variable region appears to have undergone block gene duplication and likely deletion at regular breakpoints, suggestive of a site-specific mechanism. Phylogenetic analysis using complete gene sequences provided evidence of allelic diversification via gene conversion, with breakpoints between each of the extracellular domains that were associated with high guanine-cytosine (GC) content. Advancing our knowledge of cattle $M H C$ class I evolution will help inform investigations of cattle genetic diversity and disease resistance.
\end{abstract}

Keywords MHC class I C Cattle $\cdot$ Bovine $\cdot$ Evolution · Antigen presentation

John A. Hammond

john.hammond@pirbright.ac.uk

1 Livestock Viral Diseases Programme, The Pirbright Institute, Ash Road, Pirbright, Woking, Surrey GU24 0NF, UK

\section{Brief communication}

Major histocompatibility complex (MHC) class I genes encode transmembrane glycoproteins which present peptides from intracellular proteins on the cell-surface. These peptide/ MHC complexes are recognised by receptors on CD8+ T-cells and natural killer (NK) cells, and thus play a central role in cell-mediated immune responses. Allelic diversity and variable gene content are important functional characteristics of MHC genes, believed to be primarily driven by selection from rapidly evolving pathogens (Doxiadis et al. 2011; Kelley et al. 2005; Parham et al. 1995). As a consequence, the MHC locus can differ substantially in gene content and organisation even between closely related species (Kelley et al. 2005). In cattle, there are six described polymorphic classical class I (MHC class Ia) genes (Hammond et al. 2012) encoded within the MHC on chromosome 23, all of which encode functional antigen-presenting transmembrane proteins (Gaddum et al. 2003; Graham et al. 2008; Guzman et al. 2010; Guzman et al. 2008; MacHugh et al. 2009). As well as allelic polymorphism, cattle MHC class I haplotypes also vary in gene content; only one to three of these genes are present on any given haplotype, with Gene 2 being the most ubiquitous (Birch et al. 2006; Codner et al. 2012; Ellis et al. 1999).

The allelic diversity of Holstein cattle MHC class $I$ has been well studied; however, very little is known about the full gene sequences and underlying genomic structure between the numerous different haplotypes. The cattle genome contains the most complete full haplotype sequence, assembled using DNA sequence from a Hereford cow (L1 Dominette 01449) and her sire (L1 Domino 99375) (Elsik et al. 2009). This extended MHC class I region was described by Birch et al. (2008) and reviewed by Ellis and Hammond (2014). Briefly, the genome contains two MHC class Ia genes, a Gene 2 and a Gene 5. Centromeric are five pseudogenes and the non 
classical gene $\mathrm{NCl}$. However, considering the heterozygous and repetitive nature of the $\mathrm{MHC}$ and that there may be three haplotypes in the assembled data, this region of the genome assembly is considered putative. This is particularly evident at the telomeric end, as both current assemblies of the same cattle genome data differ in gene position and content (Ellis and Hammond 2014). The only other haplotype to be structurally characterised is the Holstein A14 haplotype which was mapped using a bacterial artificial chromosome (BAC) library from a homozygous dairy bull (Di Palma et al. 2002). This haplotype contains three MHC class Ia genes (5' to 3': Gene 2, Gene 4, and Gene 1) and a possible fourth gene (historically referred to as Gene " $Z$ "). To compare these divergent haplotypes and begin to assess how cattle MHC class I has evolved, we refined the Hereford MHC class I genome assembly and assembled the Holstein A14 haplotype using modern sequencing and bioinformatics methods.

To refine the Hereford genome MHC class Ia region, we exploited L1 Domino BAC clones that were previously sequenced as part of the cattle genome project (Elsik et al. 2009). Four fully sequenced and largely assembled clones which overlap the $M H C$ class Ia locus were identified: CH240-252J17, CH240-271N5, CH240-103G5, and CH240-463M1 (GenBank: FQ482148, FO681480, FQ482089, and AC182900, respectively). Our analysis of these clones using the original raw Sanger sequencing reads confirmed their overall assembly but identified base pair calling errors at the end of the clones that created some false single nucleotide polymorphisms (SNPs) and indels. For clone CH240-463M1, we could not improve on the current de novo assembly of nine unordered contigs. Based on sequence identity to the three complete clones, we were able to order these nine contigs with high confidence. Gaps between each contig ranged from 1 to $4610 \mathrm{bp}$ suggesting that relatively little sequence information was missing. Comparison between all four clones revealed an identical $12.6-\mathrm{kb}$ overlap between CH240-252J17 and CH240-271N5, and an almost identical 72.3-kb overlap between $\mathrm{CH} 240-463 \mathrm{M} 1$ and $\mathrm{CH} 240-103 \mathrm{G} 5$, except for ten positions within $200 \mathrm{bp}$ of the contig ends. The 46.5-kb overlapping region between $\mathrm{CH} 240-463 \mathrm{M} 1$ and CH240-271N5/252J17 was also almost identical (including the Gene 2 locus) except for a 514-bp inversion, two significant insertion/deletions (798 and $201 \mathrm{bp}$ ) and 56 SNPs. However, all of these differences were located within $2 \mathrm{~kb}$ of the clone ends and were almost all previously identified by our analysis of the raw sequence data as incorrectly annotated. Consequently, we are confident that these four clones represent a single MHC class I haplotype.

A comparison between the current genome builds (Btau 4.1 and UMD 3.1) and the assembled BAC clones reveals that the current assemblies, although broadly correct, likely contain some significant errors (Fig. 1). Although the order of the MHC class $I$ genes and pseudogenes is mostly correct, the number and size of several pseudogene loci and intergenic intervals are substantially different, particularly between $\mathrm{NCl}$ and Gene 5. Of note is pseudogene 3 which is a putatively functional gene in our assembly, although the large intron 1 and 2 sequences (2853 and $1971 \mathrm{bp}$, respectively) are likely to impact negatively on expression. We also found no evidence of TRIM26 pseudogenes between Gene 2 and Gene 5, and a less gene dense region telomeric of Gene 2 that only contains functional TRIM genes. It is likely that the sequence data from more than one haplotype has been incorrectly incorporated into the genome assemblies creating assembly and annotation problems.

To sequence a complete Holstein A14 MHC class Ia haplotype, the same five BAC clones from the previous study (Di Palma et al. 2002) were isolated, expanded overnight, and BAC DNA was harvested using the Qiagen Large Construct Kit (Qiagen, GmbH, Hilden, Germany). Purified BAC DNA from four of the clones (108G12, 307D8, 251E3, and 370E1) was sequenced using an Illumina MiSeq with $250 \times 250 \mathrm{bp}$ paired-end reads prepared using the Nextera Mate Pair Sample Preparation Kit (gel-free method) (Source Biosciences, Inc. Nottingham, UK) and de novo assembled using Velvet (Zerbino and Birney 2008). The remaining clone (175E3) was sequenced using the Pacific Biosciences RSII platform (mean read length $>8 \mathrm{~kb}$ ) (GATC Biotech AG, Koblenz, Germany). Read filtering and assembly was conducted using the Pacific Biosciences SMRT Analysis software (v2.3.0; http://www.pacb.com/devnet/). Gene content was queried using BLAST (Altschul et al. 1990) against GenBank, all 114 known cattle MHC class Ia and MHC class $I b$ cDNA alleles contained within the Immuno Polymorphism Database (IPD-MHC; Release 1.3.0 (18 Nov. 2011)); http://www.ebi.ac.uk/ipd/mhc/bola; (Robinson et al. 2013), and all of the genes in the extended $M H C$ class $I$ region in the genome assemblies. The final haplotype was annotated using Artemis (Rutherford et al. 2000) and has been submitted to GenBank (accession number KT005248).

To confirm our haplotype assembly, we compared an in silico-derived HindIII restriction fragment profile for each assembled BAC clone, to the previous BAC clone mapping conducted by Southern blot hybridization of HindIII fragments (Di Palma 1999). All clones matched the previously observed profiles including a discrepancy between the overlapping clones 251E3 and 370E1. These clones differ in length between the two most 3' HindIII fragments, suggesting possible heterozygosity at this end of the locus. These discordant BACs are identical over approximately $50 \mathrm{~kb}$, but there are 163 SNPs and 16 indels over the 20.4-kb overlap at the $3^{\prime}$ end. Of these, 23 SNPs lie within the coding region of $N C 1$, of which 17 are non-synonymous, with both sequences matching the previously known alleles $N C 1^{*} 00501$ and $N C 1^{*} 00601$. In contrast, comparison of $370 \mathrm{E} 1$ with our Hereford BAC 
Fig. 1 Comparison of previously published cattle genome assemblies (Birch et al. 2008;

Ellis and Hammond 2014) with the assembly in this study. Gaps within each assembly are indicated by broken lines. Closed boxes represent putatively functional genes, open boxes (and $R P L 35 a \psi$ ) represent pseudogenes. BACs used for the current analyses are shown at left

Fig. 2 Sequence identity comparison of the assembled Hereford genome "Domino" and A14 cattle $M H C$ class I regions using DOTTER (Sonnhammer and Durbin 1995) and a sliding window size of $200 \mathrm{bp}$. Putatively functional genes are shown with closed arrows, while pseudogenes are shown with open arrows. Arrows point in the direction of transcription. The positions of the BAC clones used for each assembly are indicated by each haplotype. Gaps in the CH240-463M1 assembly are indicted by broken lines and are not to scale. There are three additional contigs from this clone which are not shown due to their small $(\sim 1 \mathrm{~kb})$ size
Revised assembly based on BACs derived from Domino (BACs shown at left)

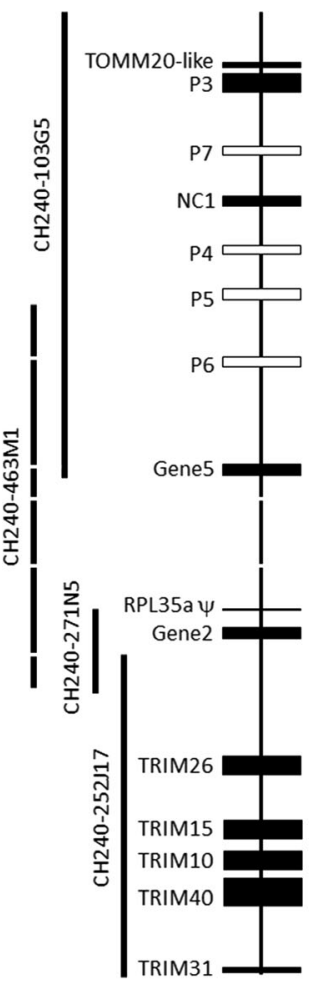

Btau 3.1 (Adapted from Birch et al. 2008)

Btau 4.6.1

(Adapted from

Ellis and Hammond 2014)
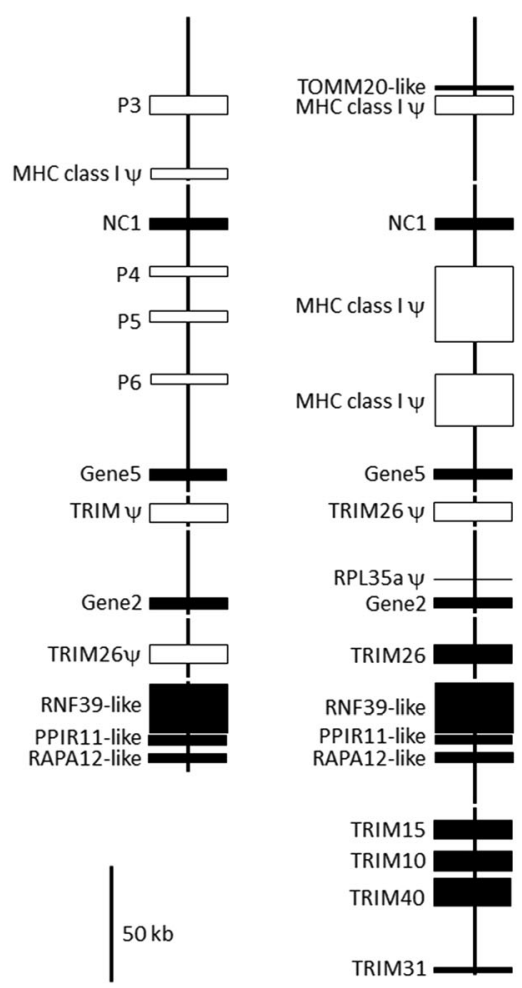

UMD 3.1

(Adapted from Ellis and Hammond 2014)
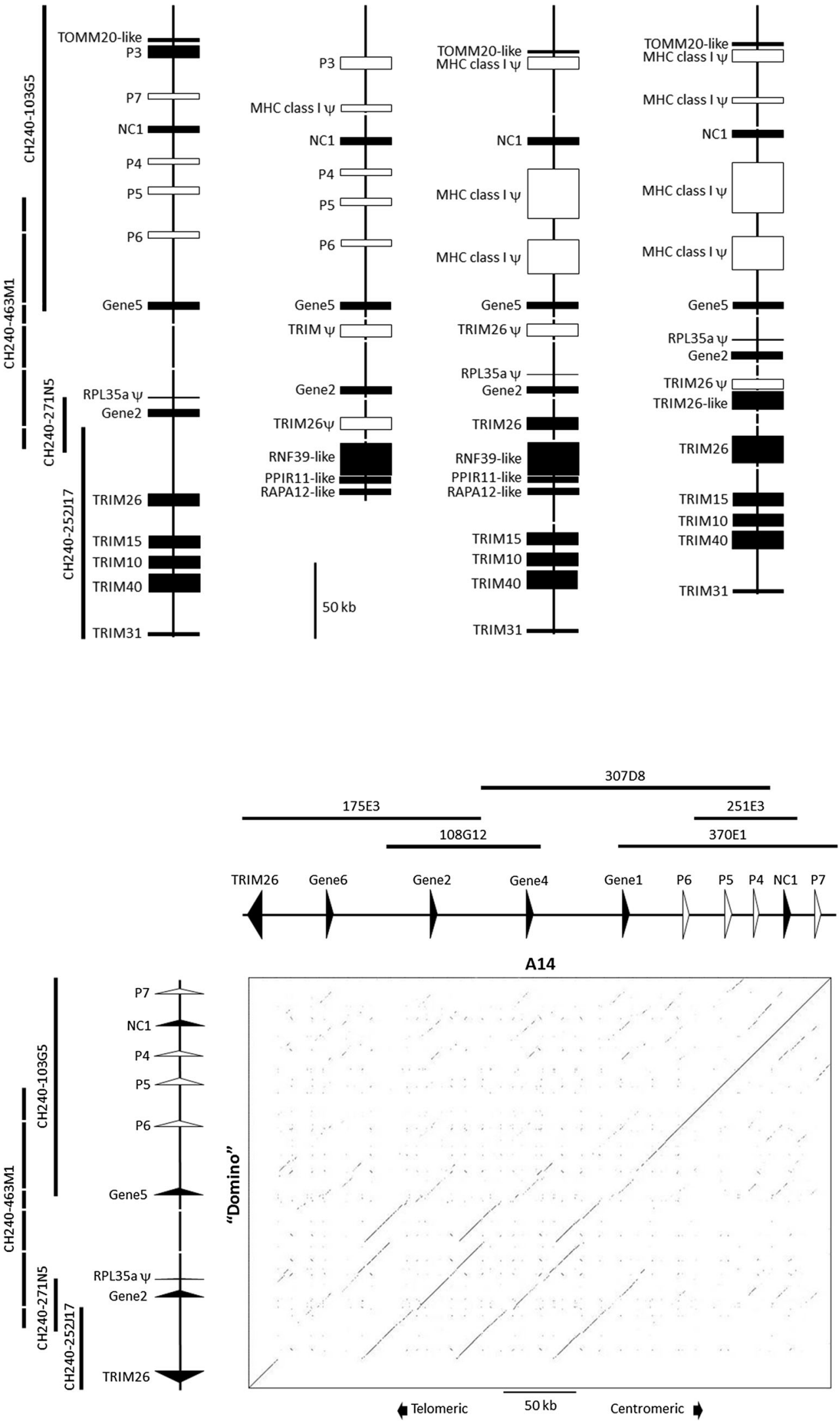
assembly reveals only 15 SNPs over approximately $98 \mathrm{~kb}$ of overlap, none of which lie within the coding region of $N C 1$. Thus, these animals from different breeds both carry $N C 1 * 00501$, with the Holstein animal being heterozygous and also carrying $N C 1^{*} 00601$.

Detailed annotation of our A14 assembly confirmed the four previously mapped $M H C$ class Ia genes upstream from $\mathrm{NCl}$ and four MHC class I-like pseudogenes (Fig. 2). All four MHC class Ia genes are separated by between 63 and $68 \mathrm{~kb}$.
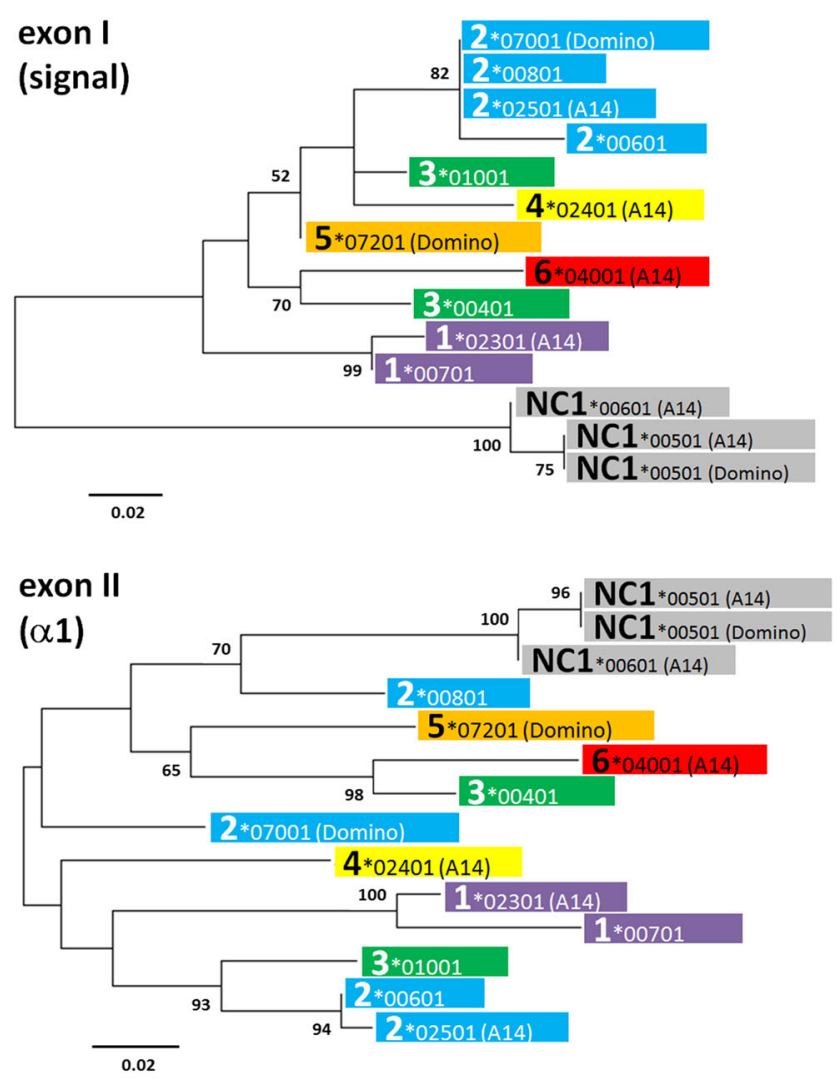

exon III

$(\alpha 2)$

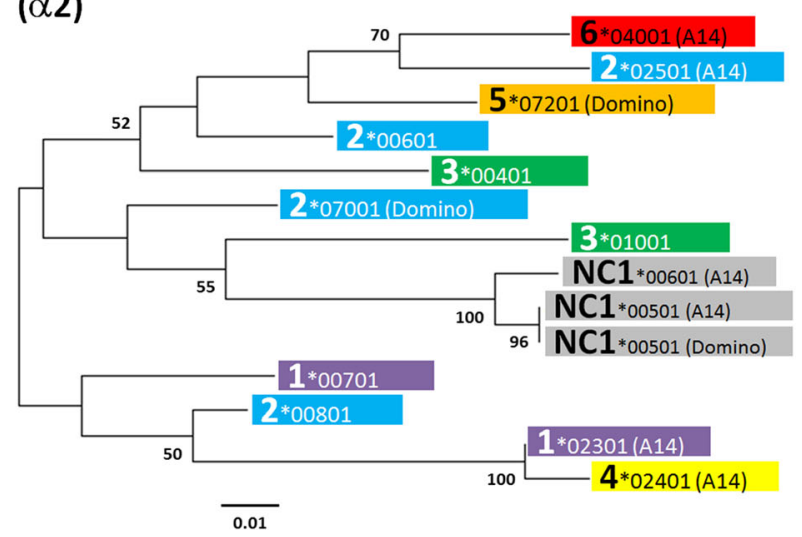

Fig. 3 Phylogenetic relationships of cattle MHC class I sequences for the first three exons and introns. Genomic sequences from A14 and Hereford genome BAC clones and from GenBank $(3 * 00401$ (AF396750), $1 * 00701$ (AF396751), 2*00601 (AF396752), 2*00801 (AF396753),
Initially, the most upstream of these was named Gene " $Z$ " and later designated $6^{*} 04001$ based on phylogenetic evidence using cDNA sequence (Di Palma et al. 2002; Hammond et al. 2012). Although direct functional evidence for $6^{*} 04001$ has not been published, mRNA expression has been confirmed by several studies. The A14 haplotype had previously been designated a three-gene haplotype, this data strongly suggests that the A14 haplotype possesses four functional MHC class Ia genes.
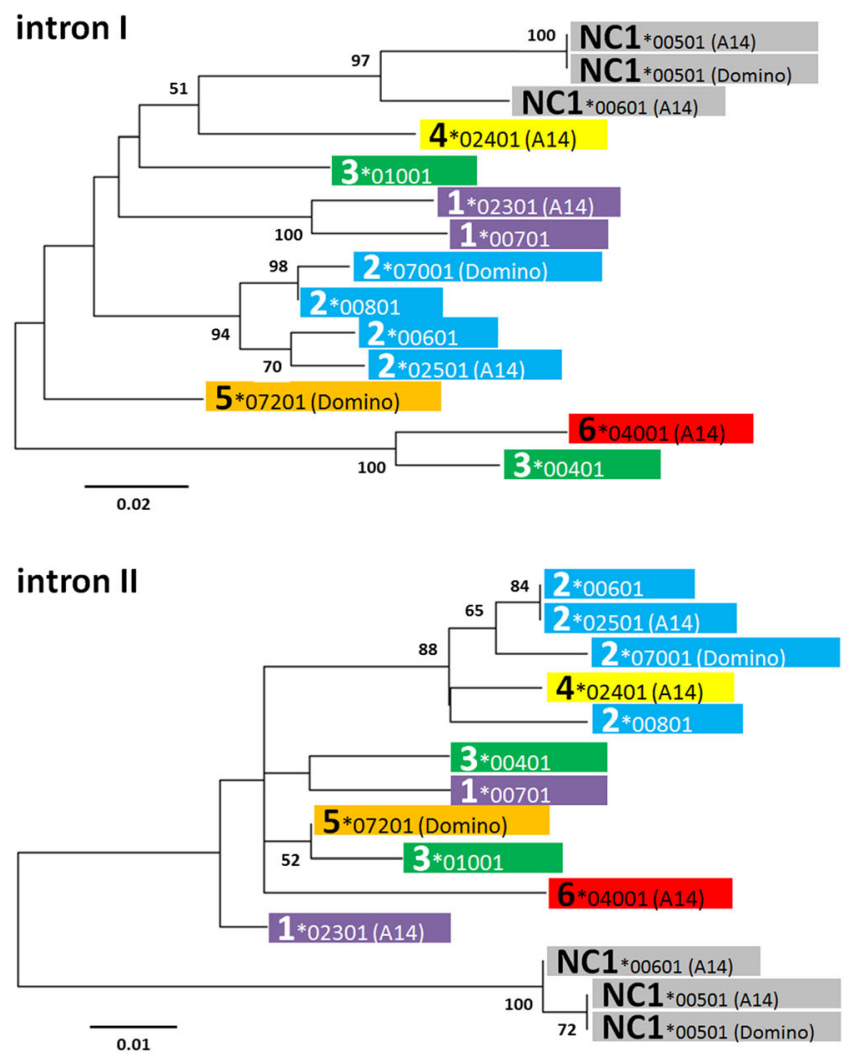

intron III

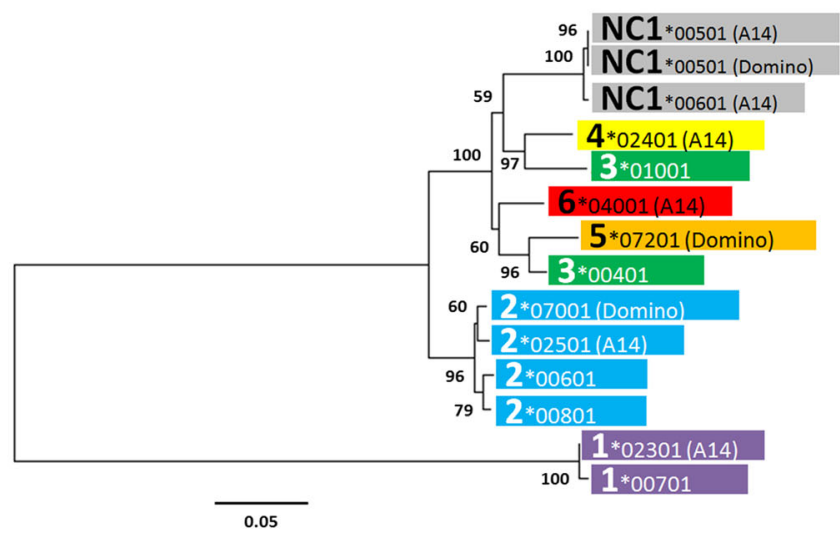

and $3 * 01001$ (AF396754)) were aligned using CLUSTAL W (Thompson et al. 1994). The tree was constructed using maximum likelihood based on the Tamura three-parameter model (Tamura 1992) within MEGA6 (Tamura et al. 2013) 
To compare the Hereford genome and the A14 haplotype, a dot-matrix plot of sequence identities across both haplotypes was generated using DOTTER (Sonnhammer and Durbin 1995). Although these are different haplotypes from different breeds, there was very little sequence variation between them

\section{NC1*00601}

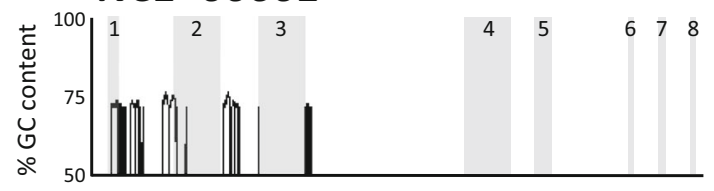

NC1 $* 00501$

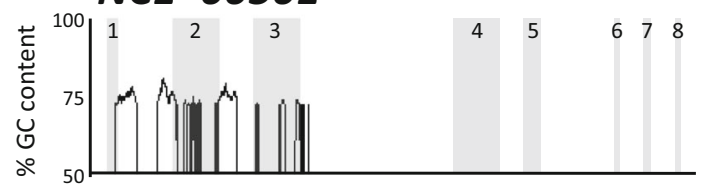

$1 * 02301$

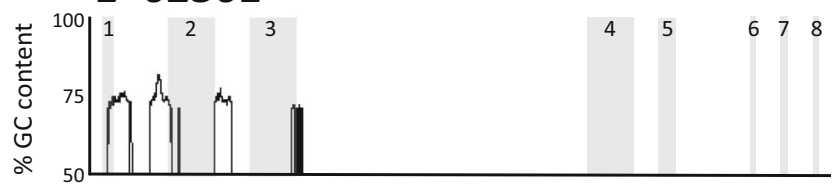

$4 * 02401$
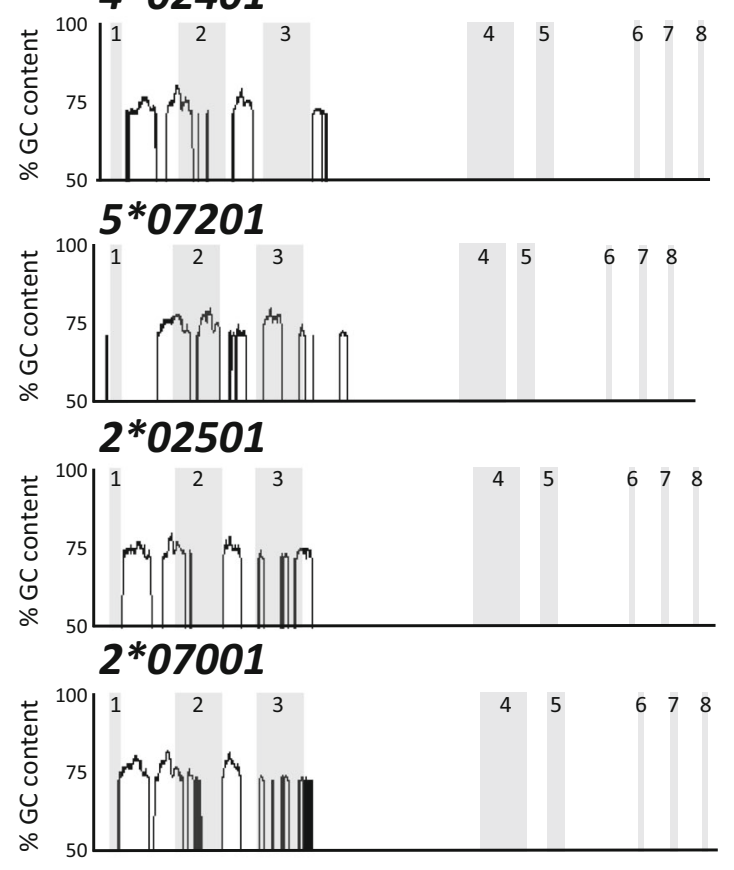

$6 * 04001$

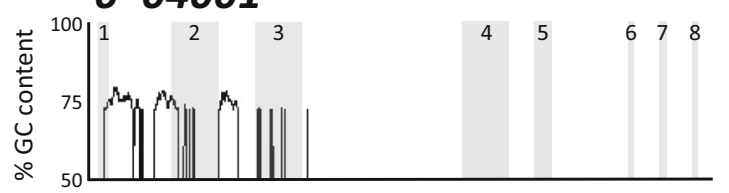

Fig. 4 Intron/exon structure and GC content of the known complete MHC class I genes. Exons for each gene are shaded and numbered. Yaxis shows percent GC (from 50 to $100 \%$ ) using a sliding window of $100 \mathrm{bp}$ and a 2.5 -standard deviation cut-off indicating abnormally high GC content near the intron/exon boundaries of the first three exons. GC content was visualised using Artemis (Rutherford et al. 2000) either side of the $M H C$ class Ia gene region, with $\mathrm{NCl}$ and all the pseudogenes being syntenic. The little variation observed was the processed (i.e. retrotransposed) pseudogene of RPL35a located downstream from Gene 2; however, no trace of this pseudogene was identifiable in the A14 data (Figs. 1 and 2). None of the class I pseudogenes described here showed any significant identity to Gene 3, the only MHC class Ia gene not present on either haplotype. Each MHC class Ia gene is located within a tandemly duplicated block of $\sim 80 \mathrm{~kb}$ that each share significant sequence identity (Fig. 2). The regular, repeating nature of these similar sized blocks is suggestive of a site-specific mechanism that has facilitated cattle MHC class I expansion and contraction. Based on these two divergent haplotypes, this recombination appears to have occurred in a distinct region between pseudogene 6 and TRIM26.

Finally, we compared the intronic and exonic sequences for all MHC class Ia and class $I b$ genes for which the information is available. This includes those from this manuscript, as well as five additional genes from GenBank; including two alleles of Gene 3 (AF396750 and AF396754), two additional alleles of Gene 2 (AF396752 and AF396753), and another allele of Gene 1 (AF396751). Domain by domain analysis showed that sequences from the current six gene designations clade together at the more conserved $3^{\prime}$ end of the gene, after the $\alpha 2$ domain in intron 3, and confirmed the close relationship of $\mathrm{NCl}$ with the classical genes (Fig. 3). The relationship between the peptide-binding domains is far more complex, with no consistent relationships emerging with either exon or intron sequence at the $5^{\prime}$ end of the molecule, apart from the $N C 1$ sequences which always form a discrete cluster. From this limited number of sequences, it is not possible to disentangle the relative contributions of mutation and recombination to this diversification. However, it seems likely that break points in introns 1,2 , and at the $5^{\prime}$ of intron 3 have led to multiple gene conversion events. Interestingly, these regions all contain unusually high guanine-cytosine (GC) content (Fig. 4), possibly representing a signature of GC-biased gene conversion (Mancera et al. 2008). As well as gene conversion, diversity would be further facilitated by the haplotype block structure that the classical genes have formed.

In conclusion, this study presents the cattle genome $M H C$ class I haplotype with more confidence and compares this to the first fully sequenced haplotype. We confirm the general organisation of the $M H C$ class I region and have identified a haplotype structure and regions of recombination that point to the mechanisms driving diversity. These results will enable more detailed studies into the evolution and the functional consequences of this diversity across a range of haplotypes and breeds. 
Acknowledgements This work was funded by the United Kingdom Biotechnology and Biological Sciences Research Council Institute Strategic Program on Livestock Viral Diseases awarded to The Pirbright Institute.

Open Access This article is distributed under the terms of the Creative Commons Attribution 4.0 International License (http:// creativecommons.org/licenses/by/4.0/), which permits unrestricted use, distribution, and reproduction in any medium, provided you give appropriate credit to the original author(s) and the source, provide a link to the Creative Commons license, and indicate if changes were made.

\section{References}

Altschul SF, Gish W, Miller W, Myers EW, Lipman DJ (1990) Basic local alignment search tool. J Mol Biol 215:403-410

Birch J, Murphy L, MacHugh ND, Ellis SA (2006) Generation and maintenance of diversity in the cattle MHC class I region. Immunogenetics 58:670-679

Birch J, Codner G, Guzman E, Ellis SA (2008) Genomic location and characterisation of nonclassical MHC class I genes in cattle. Immunogenetics 60:267-273

Codner GF, Birch J, Hammond JA, Ellis SA (2012) Constraints on haplotype structure and variable gene frequencies suggest a functional hierarchy within cattle MHC class I. Immunogenetics 64:435-445

Di Palma F (1999) Analysis and mapping of bovine MHC class I genes. Doctoral thesis. University of Reading, Reading, UK

Di Palma F, Archibald SD, Young JR, Ellis SA (2002) A BAC contig of approximately $400 \mathrm{~kb}$ contains the classical class I major histocompatibility complex (MHC) genes of cattle. Eur J Immunogenet 29: 65-68

Doxiadis GG, de Groot N, Otting N, Blokhuis JH, Bontrop RE (2011) Genomic plasticity of the MHC class I A region in rhesus macaques: extensive haplotype diversity at the population level as revealed by microsatellites. Immunogenetics 63:73-83

Ellis SA, Hammond JA (2014) The functional significance of cattle major histocompatibility complex class I genetic diversity. Annu Rev Anim Biosci 2:285-306

Ellis SA, Holmes EC, Staines KA, Smith KB, Stear MJ, McKeever DJ, MacHugh ND, Morrison WI (1999) Variation in the number of expressed MHC genes in different cattle class I haplotypes. Immunogenetics 50:319-328

Elsik CG, Tellam RL, Worley KC, Gibbs RA, Muzny DM, Weinstock GM, Adelson DL, Eichler EE, Elnitski L, Guigo R, Hamernik DL, Kappes SM, Lewin HA, Lynn DJ, Nicholas FW, Reymond A, Rijnkels M, Skow LC, Zdobnov EM, Schook L, Womack J, Alioto T, Antonarakis SE, Astashyn A, Chapple CE, Chen HC, Chrast J, Camara F, Ermolaeva O, Henrichsen CN et al (2009) The genome sequence of taurine cattle: a window to ruminant biology and evolution. Science 324:522-528
Gaddum RM, Cook RS, Furze JM, Ellis SA, Taylor G (2003) Recognition of bovine respiratory syncytial virus proteins by bovine CD8+ T lymphocytes. Immunology 108:220-229

Graham SP, Pelle R, Yamage M, Mwangi DM, Honda Y, Mwakubambanya RS, de Villiers EP, Abuya E, Awino E, Gachanja J, Mbwika F, Muthiani AM, Muriuki C, Nyanjui JK, Onono FO, Osaso J, Riitho V, Saya RM, Ellis SA, McKeever DJ, MacHugh ND, Gilbert SC, Audonnet JC, Morrison WI, van der Bruggen P, Taracha EL (2008) Characterization of the fine specificity of bovine CD8 T-cell responses to defined antigens from the protozoan parasite Theileria parva. Infect Immun 76:685-694

Guzman E, Taylor G, Charleston B, Skinner MA, Ellis SA (2008) An MHC-restricted CD8+ T-cell response is induced in cattle by footand-mouth disease virus (FMDV) infection and also following vaccination with inactivated FMDV. J Gen Virol 89:667-675

Guzman E, Taylor G, Charleston B, Ellis SA (2010) Induction of a crossreactive $\mathrm{CD} 8(+) \mathrm{T}$ cell response following foot-and-mouth disease virus vaccination. J Virol 84:12375-12384

Hammond JA, Marsh SG, Robinson J, Davies CJ, Stear MJ, Ellis SA (2012) Cattle MHC nomenclature: is it possible to assign sequences to discrete class I genes? Immunogenetics 64:475-480

Kelley J, Walter L, Trowsdale J (2005) Comparative genomics of major histocompatibility complexes. Immunogenetics 56:683-695

MacHugh ND, Connelley T, Graham SP, Pelle R, Formisano P, Taracha EL, Ellis SA, McKeever DJ, Burrells A, Morrison WI (2009) CD8+ T-cell responses to Theileria parva are preferentially directed to a single dominant antigen: Implications for parasite strain-specific immunity. Eur J Immunol 39:2459-2469

Mancera E, Bourgon R, Brozzi A, Huber W, Steinmetz LM (2008) Highresolution mapping of meiotic crossovers and non-crossovers in yeast. Nature 454:479-485

Parham P, Adams EJ, Arnett KL (1995) The origins of HLA-A, B, C polymorphism. Immunol Rev 143:141-180

Robinson J, Halliwell JA, McWilliam H, Lopez R, Marsh SG (2013) IPD - the immuno polymorphism database. Nucleic Acids Res 41: D1234-D1240

Rutherford K, Parkhill J, Crook J, Horsnell T, Rice P, Rajandream MA, Barrell B (2000) Artemis: sequence visualization and annotation. Bioinformatics 16:944-945

Sonnhammer EL, Durbin R (1995) A dot-matrix program with dynamic threshold control suited for genomic DNA and protein sequence analysis. Gene 167:GC1-GC10

Tamura K (1992) Estimation of the number of nucleotide substitutions when there are strong transition-transversion and $\mathrm{G}+\mathrm{C}$-content biases. Mol Biol Evol 9:678-687

Tamura K, Stecher G, Peterson D, Filipski A, Kumar S (2013) MEGA6: molecular evolutionary genetics analysis version 6.0. Mol Biol Evol 30:2725-2729

Thompson JD, Higgins DG, Gibson TJ (1994) CLUSTAL W: improving the sensitivity of progressive multiple sequence alignment through sequence weighting, position-specific gap penalties and weight matrix choice. Nucleic Acids Res 22:4673-4680

Zerbino DR, Birney E (2008) Velvet: algorithms for de novo short read assembly using de Bruijn graphs. Genome Res 18:821-829 